\title{
Pulsar-wind nebulae in X-rays and TeV $\gamma$-rays
}

\author{
O. Kargaltsev* and G. G. Pavlov ${ }^{\dagger}$ \\ *University of Florida, Bryant Space Center, Gainesville, FL 32611, USA \\ ${ }^{\dagger}$ Pennsylvania State University, 525 Davey Lab., University Park, PA 16802, USA
}

\begin{abstract}
Pulsars are known to be efficient accelerators that produce copious amounts of relativistic particles and inject them into the Galactic medium. The radiation emitted by such a pulsar wind can be seen from radio through $\gamma$-rays as a pulsar-wind nebula (PWN). Here we overview and summarize recent progress in X-ray and $\mathrm{TeV}$ observations of PWNe.
\end{abstract}

Keywords: Pulsar Wind Nebulae; Pulsars; Supernova Remnants; Neutron Stars

PACS: 97.60.Gb; 98.38.Mz; 98.38.-j; 98.70.Qy; 97.60.Jd

Along with supernovae and microquasars, pulsars are among the primary sources of the leptonic cosmic rays. The energies of the pulsar wind electrons and positrons range from $\lesssim 1 \mathrm{GeV}$ to $\sim 1 \mathrm{PeV}$, placing their synchrotron and inverse Compton (IC) emission into radio- $\mathrm{X}$-ray and $\mathrm{GeV}-\mathrm{TeV}$ bands, respectively. This multiwavelength emission can be seen as a pulsar-wind nebula (PWN; see [1] and [2] for reviews). During the last decade, observations with modern $\mathrm{X}$-ray and $\mathrm{TeV}$ observatories have dramatically increased the number of known PWNe whose properties are summarized in this review.

As of December 2009, about 60 PWNe associated with known radio or $\gamma$-ray pulsars have been detected. We have compiled the properties of these PWNe and their host pulsars in Tables 1 and 2. In most cases we have reanalyzed the X-ray PWN properties to ensure the uniformity of the analysis. The luminosities, spectral slopes, and sizes of the TeV PWNe have been taken from publications as well as from recent conference presentations. Because of the page limit, we mainly discuss the luminosities and, very briefly, spectra and sizes of these PWNe.

We see from Table 2 that the TeV PWN sizes generally increase with pulsar age while the X-ray PWN sizes ${ }^{1}$ show an opposite trend. Moreover, for pulsars older than $\sim 10 \mathrm{kyr}$ the sizes of the TeV PWNe are typically 100-1000 times larger than the sizes of the X-ray PWNe, while the difference is only a factor of a few for some younger pulsars (e.g., Crab). This suggests that the aged and cooled electrons (mainly seen through their synchrotron emission in radio and IC emission in $\mathrm{TeV}$ ) have propagated, through advection and/or diffusion, farther away from the pulsar than the recently injected electrons, responsible for the X-ray synchrotron nebulae. While comparing the $\mathrm{X}$-ray and $\mathrm{TeV} \mathrm{PWNe}$, one should take into account that larger numbers of electrons have been produced earlier in the pulsar's life (the electron production rate $\propto \dot{E}(t) \propto$ $\dot{E}_{0}\left(1+t / \tau_{0}\right)^{-(n+1) /(n-1)}$, where $n$ is the pulsar's braking index and $\tau_{0}$ is the initial spin-

\footnotetext{
${ }^{1}$ Here, while referring to X-ray PWN size, spectrum, or luminosity, we only consider the bright PWN "core" restricted to the torus/arcs regions or the "bullet" in ram pressure confined PWNe (see [2]).
} 
TABLE 1. Pulsars with X-ray and/or TeV PWNe

\begin{tabular}{|c|c|c|c|c|c|c|c|}
\hline$\#$ & PSR & PWN* & VHE src..$^{\dagger}$ & $\begin{array}{c}\log \dot{E} \\
{[\mathrm{erg} / \mathrm{s}]}\end{array}$ & $\begin{array}{l}\log \tau \\
{[\mathrm{yr}]}\end{array}$ & $\begin{array}{l}d^{* *} \\
\mathrm{kpc}\end{array}$ & Rad. $/ \mathrm{H}_{\alpha} / \mathrm{GeV}^{\frac{1}{2}}$ \\
\hline 1 & J0537-6910 & N157B & & 38.68 & 3.70 & 50 & $\mathrm{Y} / \mathrm{N} / \mathrm{N}$ \\
\hline 2 & B0531+21 & Crab & H J0534+220 & 38.66 & 3.09 & 2 & $\mathrm{Y} / \mathrm{N} / \mathrm{Y}$ \\
\hline 3 & $\mathrm{~J} 2022+3842$ & G76.9+1.0 & $\ldots$ & 38.30 & 3.95 & 8 & $\mathrm{Y} / \mathrm{N} / \mathrm{N}$ \\
\hline 4 & B0540-69 & N158A & 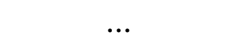 & 38.17 & 3.22 & 50 & $\mathrm{Y} / \mathrm{N} / \mathrm{N}$ \\
\hline 5 & J1813-1749 & G12.82-0.02 & H J1813-178? & 37.83 & 3.66 & 4.5 & N/N/P \\
\hline 6 & J1400-6325 & G310.6-1.6 & & 37.71 & 4.10 & 6 & $\mathrm{Y} / \mathrm{N} / \mathrm{N}$ \\
\hline 7 & J1833-1034 & G21.50-0.89 & H J1833-105 & 37.52 & 3.69 & 4.8 & $\mathrm{Y} / \mathrm{N} / \mathrm{Y}$ \\
\hline 8 & J0205+6449 & $3 \mathrm{C} 58$ & & 37.43 & 3.73 & 3.2 & $\mathrm{Y} / \mathrm{N} / \mathrm{Y}$ \\
\hline 9 & $\mathrm{~J} 2229+6114$ & G106.65+2.96 & V J2228+609 & 37.35 & 4.02 & 3 & $\mathrm{Y} / \mathrm{N} / \mathrm{Y}$ \\
\hline 10 & В1509-58 & Jellyfish & H J1514-591 & 37.25 & 3.19 & 5 & $\mathrm{P} / \mathrm{N} / \mathrm{N}$ \\
\hline 11 & $\mathrm{~J} 1617-5055$ & G332.50-0.28 & H J1616-508? & 37.20 & 3.91 & 6.5 & N/N/P \\
\hline 12 & J1124-5916 & G292.04+1.75 & $\ldots$ & 37.07 & 3.46 & 6 & $\mathrm{Y} / \mathrm{N} / \mathrm{Y}$ \\
\hline 13 & $\mathrm{~J} 1930+1852$ & G54.10+0.27 & V J1930+188 & 37.06 & 3.46 & 6.2 & $\mathrm{Y} / \mathrm{N} / \mathrm{N}$ \\
\hline 14 & J1420-6048 & G313.54+0.23 & H J1420-607 & 37.02 & 4.11 & 5.6 & $\mathrm{P} / \mathrm{N} / \mathrm{N}$ \\
\hline 15 & $\mathrm{~J} 1846-0258$ & Kes 75 & H J1846-029 & 36.91 & 2.86 & $6 ? ?$ & $\mathrm{Y} / \mathrm{N} / \mathrm{N}$ \\
\hline 16 & В0833-45 & Vela & H J0835-455 & 36.84 & 4.05 & $0.29^{\mathrm{p}}$ & $\mathrm{Y} / \mathrm{N} / \mathrm{N}$ \\
\hline 17 & J1811-1925 & G11.18-0.35 & & 36.81 & 4.37 & 5 & $\mathrm{Y} / \mathrm{N} / \mathrm{N}$ \\
\hline 18 & J1838-0655 & G25.24-0.19 & H J1837-069 & 36.74 & 4.36 & 7 & $\mathrm{~N} / \mathrm{N} / \mathrm{N}$ \\
\hline 19 & J1418-6058 & Rabbit & H J1418-609 & 36.69 & 4.00 & $3 ?$ & $\mathrm{Y} / \mathrm{N} / \mathrm{Y}$ \\
\hline 20 & $\mathrm{~J} 1856+0245$ & $\mathrm{G} 36.01+0.06^{?}$ & H J1857+026 & 36.66 & 4.32 & 9 & $\mathrm{~N} / \mathrm{N} / \mathrm{N}$ \\
\hline 21 & B1951+32 & G68.77+2.82 & $\ldots$ & 36.57 & 5.03 & 2.5 & $\mathrm{Y} / \mathrm{Y} / \mathrm{Y}$ \\
\hline 22 & J1826-1256 & Eel & $\ldots$ & 36.55 & 4.15 & $7^{?}$ & $\mathrm{P} / \mathrm{N} / \mathrm{Y}$ \\
\hline 23 & $\mathrm{~J} 2021+3651$ & G75.23+0.12 & M J2019+37? & 36.53 & 4.23 & 4 & N/N/Y \\
\hline 24 & B1706-44 & G343.10-2.69 & H J1708-443 & 36.53 & 4.24 & 2 & $\mathrm{Y} / \mathrm{N} / \mathrm{Y}$ \\
\hline 25 & J1357-6429 & G309.92-2.51 & H J1357-645 & 36.49 & 3.86 & 2.5 & $\mathrm{P} / \mathrm{N} / \mathrm{N}$ \\
\hline 26 & $\mathrm{~J} 1913+1011$ & G44.48-0.17? & H J1912+101 & 36.46 & 5.23 & 4.5 & $\mathrm{~N} / \mathrm{N} / \mathrm{N}$ \\
\hline 27 & $\mathrm{~J} 1907+0602$ & G40.16-0.89? & H J1908+063? & 36.45 & 4.28 & 3.2 & $\mathrm{~N} / \mathrm{N} / \mathrm{Y}$ \\
\hline 28 & B1823-13 & G18.00-0.69 & H J1825-137 & 36.45 & 4.33 & 4 & $\mathrm{~N} / \mathrm{N} / \mathrm{N}$ \\
\hline 29 & B1757-24 & Duck & $\ldots$ & 36.41 & 4.19 & 5 & $\mathrm{Y} / \mathrm{N} / \mathrm{N}$ \\
\hline 30 & J1016-5857 & G284.08-1.88 & $\ldots$ & 36.41 & 4.32 & 3 & $\mathrm{~N} / \mathrm{N} / \mathrm{N}$ \\
\hline 31 & J1747-2958 & Mouse & $\ldots$ & 36.40 & 4.41 & 5 & $\mathrm{Y} / \mathrm{N} / \mathrm{Y}$ \\
\hline 32 & J1119-6127 & G292.15-0.54 & H J1119-615? & 36.37 & 3.21 & 8.4 & $\mathrm{~N} / \mathrm{N} / \mathrm{N}$ \\
\hline 33 & B1800-21 & G8.40+0.15 & H J1804-216? & 36.34 & 4.20 & 4 & N/N/P \\
\hline 34 & B1046-58 & $\mathrm{G} 287.42+0.58$ & $\ldots$ & 36.30 & 4.31 & 3 & $\mathrm{~N} / \mathrm{N} / \mathrm{Y}$ \\
\hline 35 & J1809-1917 & G11.09+0.08 & H J1809-193 & 36.25 & 4.71 & 3.5 & $\mathrm{P} / \mathrm{N} / \mathrm{N}$ \\
\hline 36 & J1301-6305 & G304.10-0.24 & H J1303-631? & 36.22 & 4.04 & 7 & $\mathrm{~N} / \mathrm{N} / \mathrm{N}$ \\
\hline 37 & J1718-3825 & G348.95-0.43 & H J1718-385? & 36.11 & 4.95 & 4 & $\mathrm{~N} / \mathrm{N} / \mathrm{Y}$ \\
\hline 38 & J1531-5610 & G323.89+0.03 & $\ldots$ & 35.96 & 4.99 & 3 & $\mathrm{~N} / \mathrm{N} / \mathrm{N}$ \\
\hline 39 & J1509-5850 & G319.97-0.62 & $\ldots$ & 35.71 & 5.19 & 4 & $\mathrm{P} / \mathrm{N} / \mathrm{Y}$ \\
\hline 40 & $\mathrm{~J} 1857+0143$ & G35.17-0.57? & H J1858+020? & 35.65 & 4.85 & 5.5 & $\mathrm{~N} / \mathrm{N} / \mathrm{N}$ \\
\hline 41 & $\mathrm{~J} 0007+7303$ & CTA1 & $\ldots$ & 35.65 & 4.15 & 1.4 & N/N/Y \\
\hline 42 & B1853+01 & G34.56-0.50 & $\ldots$ & 35.63 & 4.31 & 3 & $\mathrm{Y} / \mathrm{N} / \mathrm{N}$ \\
\hline 43 & J1809-2332 & Taz & $\ldots$ & 35.60 & 4.36 & 2 & $\mathrm{Y} / \mathrm{N} / \mathrm{N}$ \\
\hline 44 & $\mathrm{~J} 1958+2846$ & G65.89-0.37? & M J1954+28 & 35.58 & 4.32 & $2 ?$ & $\mathrm{~N} / \mathrm{N} / \mathrm{Y}$ \\
\hline 45 & J1702-4128 & G344.74+0.12 & H J1702-420? & 35.53 & 4.74 & 5 & $\mathrm{~N} / \mathrm{N} / \mathrm{N}$ \\
\hline 46 & J0729-1448 & G230.39-1.42 & & 35.45 & 4.54 & 4 & $\mathrm{~N} / \mathrm{N} / \mathrm{N}$ \\
\hline 47 & $\mathrm{~J} 2032+4127$ & G80.22+1.02 & He J2032+4130 & 35.43 & 5.04 & 1.7 & $\mathrm{~N} / \mathrm{N} / \mathrm{Y}$ \\
\hline 48 & $\mathrm{~J} 1740+1000$ & $\mathrm{G} 34.01+20.27$ & $\ldots$ & 35.36 & 5.06 & 1.4 & $\mathrm{~N} / \mathrm{N} / \mathrm{N}$ \\
\hline
\end{tabular}


TABLE 1. Pulsars with X-ray and/or TeV PWNe (continued)

\begin{tabular}{|c|c|c|c|c|c|c|c|}
\hline \# & PSR & $\mathbf{P W N}^{*}$ & VHE src..$^{\dagger}$ & $\begin{array}{c}\log \dot{E} \\
{[\mathrm{erg} / \mathrm{s}]}\end{array}$ & $\begin{array}{c}\log \tau \\
{[\mathrm{yr}]}\end{array}$ & $\begin{array}{r}d^{* *} \\
\mathrm{kpc}\end{array}$ & Rad. $/ \mathrm{H}_{\alpha} / \mathrm{GeV}^{\ddagger}$ \\
\hline 49 & J0631+1036 & $\mathrm{G} 201.22+0.45^{?}$ & M J0630+10? & 35.24 & 4.64 & 3.6 & $\mathrm{~N} / \mathrm{N} / \mathrm{Y}$ \\
\hline 50 & B $1957+20$ & G59.20-4.70 & $\ldots$ & 35.20 & 9.18 & 2.5 & N/Y/N \\
\hline 51 & J0633+0632 & G205.10-0.93 & $\ldots$ & 35.08 & 4.77 & $1.5^{?}$ & N/N/Y \\
\hline 52 & J1740-3015 & G358.29+0.24? & H J1741-302 & 34.91 & 4.31 & 3 & N/N/N \\
\hline 53 & $\mathrm{~J} 0538+2817$ & G179.72-1.69 & $\ldots$ & 34.69 & 5.79 & $1.47^{\mathrm{p}}$ & N/N/N \\
\hline 54 & B $0355+54$ & Mushroom & $\ldots$ & 34.66 & 5.75 & $1.04^{\mathrm{p}}$ & N/N/N \\
\hline 55 & J0633+1746 & Geminga & M J0632+17? & 34.51 & 5.53 & $0.25^{\mathrm{p}}$ & N/N/Y \\
\hline 56 & J1745-3040 & G358.55-0.96? & H $1745-305 ?$ & 33.93 & 5.74 & 2 & N/N/N \\
\hline 57 & $\mathrm{~J} 1502-5828$ & $\mathrm{G} 319.39+0.13^{?}$ & H J1503-582? & 33.68 & 5.46 & 8 & $\mathrm{~N} / \mathrm{N} / \mathrm{N}$ \\
\hline 58 & B1929+10 & G47.38-3.88 & $\ldots$ & 33.59 & 6.49 & $0.36^{\mathrm{p}}$ & $\mathrm{P} / \mathrm{N} / \mathrm{N}$ \\
\hline 59 & B2224+65 & Guitar & $\ldots$ & 33.07 & 6.05 & 1.5 & N/Y/N \\
\hline
\end{tabular}

* PWN name or galactic coordinates. The superscript ${ }^{?}$ mark the cases in which no X-ray PWN has been reported, but there are TeV PWN candidates nearby.

$\dagger \mathrm{TeV}$ sources in the vicinity of the PSR/PWN. 'H', 'V', 'M' and 'He' stand for HESS, VERITAS, Milagro and HEGRA. The superscript ${ }^{?}$ marks questionable associations.

** Our best guess for the pulsar distance, used to scale the distance-dependent parameters in Table 2. The superscript ${ }^{\mathrm{P}}$ marks the distances determined from parallax measurements; the most uncertain distances (e.g., when even dispersion measure is unknown) are marked by ?. For Kes 75 the distance (marked by

??) is rather uncertain, ranging from 5.1-7.5 kpc ([3]) to $\sim 10.6 \mathrm{kpc}([4])$.

$\ddagger$ Is the PWN detected in radio/ $\mathrm{H}_{\alpha}$, and PSR/PWN in GeV $\gamma$-rays? $\mathrm{P}=$ 'possibly'.

down timescale; e.g., [1]). This picture is further complicated by environmental effects and pulsar motion. For instance, the ambient pressure that confines a slowly-moving PWN, is much greater in a star-forming region than in low-density regions above the Galactic plane; high-speed pulsars are accompanied by long tails with higher bulk flow velocities compared to more isotropic PWNe around slowly-moving pulsars; pressure gradients inside a supernova remnant or interaction with its reverse shock can affect the PWN shape. In addition, the ambient radio/IR radiation density may vary and affect the properties of the IC radiation emitted by the pulsar wind. In many cases, significant offsets between the centroid of the extended TeV source and the neighboring pulsar's position may be attributed to the interaction with the asymmetric reverse shock or to a locally non-uniform distribution of the ambient matter/radiation. The large sizes of these $\mathrm{TeV}$ sources and the large offsets may sometimes lead to false associations with pulsars, especially if there is no preferential extension of the X-ray PWN toward the TeV source center.

The X-ray and TeV spectra of PWNe are often approximated by power-law (PL) models with different slopes. If the electron spectral energy distribution (SED) can be approximated by a single PL, $d N(\varepsilon) \propto \varepsilon^{-p} d \varepsilon$, then both the synchrotron (X-ray) and IC $(\mathrm{TeV})$ spectra should have the same slopes, $F_{V} \propto v^{-\Gamma+1}$, where the photon index $\Gamma=(p+1) / 2$. However, one can see from Table 2 that the spectra of X-ray PWNe are systematically harder than those of their TeV counterparts, which can be attributed to the evolution of the electron SED. Indeed, Chandra observations of bright, well-resolved PWNe have shown that the synchrotron spectrum varies significantly with the distance from the pulsar (due to the radiation and expansion energy losses), and therefore a single PL approximation to the X-ray spectrum becomes inapplicable if a large enough volume 
TABLE 2. Properties of the $\mathrm{X}$-ray/TeV PWNe listed in Table 1

\begin{tabular}{|c|c|c|c|c|c|c|c|c|c|}
\hline$\#$ & $N_{\mathrm{H}, 22}{ }^{*}$ & $\begin{array}{c}\log L_{X}^{\dagger} \\
{[\mathrm{erg} / \mathrm{s}]}\end{array}$ & $\Gamma_{\mathrm{X}}$ & $\begin{array}{c}\log L_{\gamma}^{* *} \\
{[\operatorname{erg} / \mathrm{s}]}\end{array}$ & $\Gamma_{\gamma}^{\ddagger}$ & $\begin{array}{c}l_{X}^{\S} \\
\mathrm{pc}\end{array}$ & $\begin{array}{l}l_{\gamma}^{\mathrm{g}} \\
\mathrm{pc}\end{array}$ & $\begin{array}{c}\Delta^{\|} \\
\operatorname{arcmin}\end{array}$ & Refs. ${ }^{\dagger \dagger}$ \\
\hline 1 & 0.5 & $36.04 \pm 0.01$ & $2.20 \pm 0.05$ & & & 1.4 & & & [5] \\
\hline 2 & 0.32 & $37.28 \pm 0.01$ & $2.12 \pm 0.01$ & $34.51 \pm 0.06$ & $2.63 \pm 0.20$ & 1.2 & $<2$ & $<0.5$ & {$[6,7,8]$} \\
\hline 3 & 1.3 & $32.58 \pm 0.12$ & $0.9 \pm 0.5$ & $\ldots$ & $\ldots$ & 0.4 & $\ldots$ & $\ldots$ & [9] \\
\hline 4 & 0.46 & $37.01 \pm 0.01$ & $1.85 \pm 0.10$ & $\ldots$ & $\ldots$ & 1.4 & $\ldots$ & $\ldots$ & [10] \\
\hline 5 & 10 & $32.90 \pm 0.25$ & $0.4_{-0.7}^{+0.4}$ & $34.34 \pm 0.14$ & $2.09 \pm 0.21$ & 2.0 & 6 & $<0.5$ & {$[11,12,13]$} \\
\hline 6 & 2.1 & $34.99 \pm 0.04$ & $1.83 \pm 0.09$ & $\ldots$ & & 2.3 & $\ldots$ & . & [14] \\
\hline 7 & 2.3 & $35.36 \pm 0.01$ & $1.89 \pm 0.02$ & $33.63 \pm 0.12$ & $2.08 \pm 0.22$ & 1.0 & $<5$ & $<1$ & {$[15,16]$} \\
\hline 8 & 0.43 & $33.94 \pm 0.01$ & $2.02 \pm 0.01$ & $\ldots$ & $\ldots$ & 1.2 & $\ldots$ & $\ldots$ & [17] \\
\hline 9 & 0.5 & $32.94 \pm 0.01$ & $1.3 \pm 0.1$ & $33.63 \pm 0.20$ & $2.3 \pm 0.4$ & 0.4 & 26 & 24 & {$[18,19]$} \\
\hline 10 & 0.8 & $34.60 \pm 0.03$ & $1.65 \pm 0.05$ & $34.86 \pm 0.12$ & $2.27 \pm 0.2$ & 4.5 & 24 & 2.4 & {$[20,21]$} \\
\hline 11 & 3.5 & $33.79 \pm 0.02$ & $1.2 \pm 0.2$ & $34.78 \pm 0.06$ & $2.35 \pm 0.21$ & 0.6 & 60 & 10 & {$[22,12]$} \\
\hline 12 & 0.37 & $34.71 \pm 0.03$ & $1.7 \pm 0.5$ & $\ldots$ & $\ldots$ & 0.2 & $\ldots$ & $\ldots$ & [23] \\
\hline 13 & 1.9 & $34.46 \pm 0.01$ & $1.99 \pm 0.03$ & $33.88 \pm 0.20$ & $2.3 \pm 0.4$ & 1.5 & $<11$ & $<3$ & {$[24,19]$} \\
\hline 14 & 5.4 & $33.15 \pm 0.11$ & $0.5 \pm 1.2$ & $34.60 \pm 0.07$ & $2.17 \pm 0.12$ & 0.4 & 11 & 3.3 & {$[25,26]$} \\
\hline 15 & 4.0 & $35.19 \pm 0.02$ & $2.03 \pm 0.02$ & $33.87 \pm 0.09$ & $2.26 \pm 0.15$ & 2.8 & $<6$ & $<1$ & [27] \\
\hline 16 & 0.02 & $32.11 \pm 0.03$ & $1.4 \pm 0.1$ & $32.14 \pm 0.22$ & $1.45 \pm 0.22^{?}$ & 0.1 & 5 & 30 & $28,29,30]$ \\
\hline 17 & 3.1 & $34.00 \pm 0.09$ & $1.5 \pm 0.2$ & & & 1.0 & 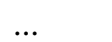 & & [31] \\
\hline 18 & 4.0 & $33.47 \pm 0.14$ & $0.8 \pm 0.3$ & $34.91 \pm 0.18$ & $2.27 \pm 0.21$ & 2.0 & 28 & 3 & {$[32,12]$} \\
\hline 19 & 2.3 & $33.55 \pm 0.02$ & $1.7 \pm 0.1$ & $33.91 \pm 0.07$ & $2.22 \pm 0.12$ & 1.5 & 8 & 3.5 & {$[25,26]$} \\
\hline 20 & $\lesssim 2$ & $\ldots$ & $\ldots$ & $35.16 \pm 0.11$ & $2.39 \pm 0.08$ & 1.7 & 40 & 6 & {$[33,34]$} \\
\hline 21 & 0.34 & $32.62 \pm 0.01$ & $1.76 \pm 0.03$ & $\ldots$ & $\ldots$ & 0.4 & $\ldots$ & $\ldots$ & {$[35,36]$} \\
\hline 22 & 2.0 & $32.41 \pm 0.05$ & $1.27 \pm 0.40$ & $\ldots$ & $\ldots$ & 0.3 & $\ldots$ & $\ldots$ & [37] \\
\hline 23 & 0.7 & $33.08 \pm 0.07$ & $1.7 \pm 0.3$ & $33.09 \pm 0.50$ & [2.6] & 0.8 & $<140$ & 18 & {$[38,39]$} \\
\hline 24 & 0.5 & $32.58 \pm 0.02$ & $1.8 \pm 0.1$ & $33.87 \pm 0.13$ & $2.00 \pm 0.22$ & 0.2 & 23 & 14 & {$[40,41]$} \\
\hline 25 & 0.23 & $31.70 \pm 0.07$ & $1.28 \pm 0.21$ & $33.77 \pm 0.20$ & $2.2 \pm 0.2$ & 0.1 & 20 & 8 & [42] \\
\hline 26 & $\lesssim 2$ & $<31.22$ & $\ldots$ & $34.19 \pm 0.19$ & $2.7 \pm 3.6$ & $\ldots$ & 60 & 12 & [43] \\
\hline 27 & $\lesssim 2$ & $\ldots$ & $\ldots$ & $34.22 \pm 0.22$ & $2.10 \pm 0.21$ & $\ldots$ & 37 & 16 & [44] \\
\hline 28 & 1.0 & $32.50 \pm 0.05$ & $1.3 \pm 0.4$ & $35.05 \pm 0.10$ & $2.26 \pm 0.2 ?$ & 0.2 & 70 & 10 & {$[45,46,47]$} \\
\hline 29 & 4.4 & $33.20 \pm 0.14$ & $2.5 \pm 0.3$ & $\ldots$ & $\ldots$ & 0.5 & $\ldots$ & $\ldots$ & [48] \\
\hline 30 & {$[1.2]$} & $32.30 \pm 0.11$ & $1.5 \pm 0.2$ & $\ldots$ & $\ldots$ & 0.1 & $\ldots$ & $\ldots$ & [49] \\
\hline 31 & 3.0 & $34.70 \pm 0.05$ & $2.0 \pm 0.2$ & $\ldots$ & $\ldots$ & 0.5 & $\ldots$ & $\ldots$ & [50] \\
\hline 32 & 1. & 3 & $1.5 \pm$ & $34.20 \pm 0$ & $>2$ & 0.5 & 30 & 6 & {$[51,52]$} \\
\hline 33 & 1.4 & $32.20 \pm 0.05$ & $1.6 \pm 0.3$ & $34.30 \pm 0.08$ & $2.72 \pm 0.21$ & 0.2 & 58 & 10 & {$[53,54,12]$} \\
\hline 34 & {$[0.4]$} & $31.82 \pm 0.04$ & $1.0 \pm 0.2$ & $\ldots$ & $\ldots$ & 0.2 & $\ldots$ & $\ldots$ & [55] \\
\hline 35 & 0.7 & $32.59 \pm 0.03$ & $1.4 \pm 0.1$ & $34.29 \pm 0.11$ & $2.20 \pm 0.22$ & 0.2 & 40 & 8 & {$[56,57]$} \\
\hline 36 & [1.1] & $32.16 \pm 0.50$ & $\ldots$ & $34.76 \pm 0.08$ & $2.44 \pm 0.21$ & 2.0 & 39 & 11 & [58] \\
\hline 37 & 0.7 & $32.60 \pm 0.10$ & $1.9 \pm 0.2$ & $33.74 \pm 0.30$ & $0.7 \pm 0.6^{?}$ & 2.0 & 7 & 3 & {$[59,57]$} \\
\hline 38 & $\lesssim 2$ & $31.03 \pm 0.5$ & $\ldots$ & $\ldots$ & $\ldots$ & 0.1 & $\ldots$ & $\ldots$ & $\ldots$ \\
\hline 39 & 2.1 & $32.12 \pm 0.13$ & $1.8 \pm 0.3$ & $\ldots$ & $\ldots$ & 0.4 & $\ldots$ & $\ldots$ & [60] \\
\hline 40 & $\leqslant 2$ & $\ldots$ & $\ldots$ & $33.82 \pm 0.25$ & $2.17 \pm 0.23$ & $\ldots$ & $<13$ & $\ldots$ & [34] \\
\hline 41 & {$[0.3]$} & $31.38 \pm 0.20$ & $1.1 \pm 0.6$ & $\ldots$ & $\ldots$ & 0.1 & $\ldots$ & $\ldots$ & [61] \\
\hline 42 & 2.0 & $31.93 \pm 0.13$ & $2.1 \pm 1.0$ & $\ldots$ & $\ldots$ & 0.4 & $\ldots$ & $\ldots$ & {$[62]$} \\
\hline 43 & 0.3 & $31.54 \pm 0.30$ & $1.5 \pm 0.6$ & $\ldots$ & & 0.3 & $\ldots$ & $\ldots$ & \\
\hline 44 & $\lesssim 1$ & $\ldots$ & $\ldots$ & $32.00 \pm 0.50$ & {$[2.6]$} & $\ldots$ & $\ldots$ & $<40$ & [39] \\
\hline 45 & [1.1] & $31.60 \pm 0.50$ & $\ldots$ & $34.30 \pm 0.11$ & $2.31 \pm 0.23$ & 0.2 & 50 & 30 & [12] \\
\hline 46 & {$[0.3]$} & $31.20 \pm 0.50$ & $\ldots$ & .. & $\ldots$ & 0.05 & $\ldots$ & $\ldots$ & $\ldots$ \\
\hline 47 & [1.5] & $31.53 \pm 0.10$ & [1.5] & $32.97 \pm 0.09$ & $1.9 \pm 0.3$ & 0.5 & 6 & 4 & {$[63,64,65]$} \\
\hline 48 & {$[0.1]$} & $31.11 \pm 0.10$ & $1.5 \pm 0.3$ & $\ldots$ & $\ldots$ & 0.8 & $\ldots$ & $\ldots$ & {$[60]$} \\
\hline
\end{tabular}


TABLE 2. Properties of the X-ray/TeV PWNe listed in Table 1

\begin{tabular}{cccccccccc}
\hline$\#$ & $N_{\mathrm{H}, 22}{ }^{*}$ & $\begin{array}{c}\log L_{\mathrm{X}}^{\dagger} \\
{[\mathrm{erg} / \mathrm{s}]}\end{array}$ & $\Gamma_{\mathrm{X}}$ & $\begin{array}{c}\log L_{\gamma}{ }^{* *} \\
{[\mathrm{erg} / \mathrm{s}]}\end{array}$ & $\Gamma_{\gamma}^{\dagger}$ & $\begin{array}{c}l_{X}{ }^{\S} \\
\mathrm{pc}\end{array}$ & $\begin{array}{c}l_{\gamma}{ }^{\mathrm{II}} \\
\mathrm{pc}\end{array}$ & $\begin{array}{c}\Delta^{\|} \\
\operatorname{arcmin}\end{array}$ & Refs. $^{\dagger \dagger}$ \\
\hline 49 & $\lesssim 0.6$ & $<31.15$ & $\ldots$ & $32.64 \pm 0.50$ & {$[2.6]$} & $\ldots$ & $<180$ & $\ldots$ & {$[39,66]$} \\
50 & 0.1 & $30.81 \pm 0.18$ & $1.6 \pm 0.5$ & $\ldots$ & $\ldots$ & 0.1 & $\ldots$ & $\ldots$ & {$[67]$} \\
51 & $\lesssim 0.04$ & $\ldots$ & $\ldots$ & $\ldots$ & $\ldots$ & 1.5 & $\ldots$ & $\ldots$ & {$[68]$} \\
52 & $\lesssim 1.5$ & $\ldots$ & $\ldots$ & $32.82 \pm 0.15$ & $2.78 \pm 0.31$ & $\ldots$ & 22 & 12 & {$[69]$} \\
53 & 0.25 & $31.04 \pm 0.10$ & $3.3 \pm 0.5$ & $\ldots$ & $\ldots$ & 0.2 & $\ldots$ & $\ldots$ & {$[70,71]$} \\
54 & 0.6 & $31.19 \pm 0.03$ & $1.5 \pm 0.1$ & $\ldots$ & $\ldots$ & 0.1 & $\ldots$ & $\ldots$ & {$[72]$} \\
55 & 0.03 & $29.71 \pm 0.07$ & $1.0 \pm 0.2$ & $30.22 \pm 0.50$ & {$[2.6]$} & 0.02 & 10 & $<30$ & {$[73,39]$} \\
56 & $\lesssim 1.3$ & $<31$ & $\ldots$ & $\sim 33.01$ & $1.82 \pm 0.35$ & $\ldots$ & 6 & 7 & {$[69]$} \\
57 & $\lesssim 2$ & $\ldots$ & $\ldots$ & $34.66 \pm 0.60$ & $2.4 \pm 0.44$ & $\ldots$ & 70 & $\ldots$ & {$[69]$} \\
58 & 0.17 & $29.63 \pm 0.01$ & $1.7 \pm 0.6$ & $\ldots$ & $\ldots$ & 0.05 & $\ldots$ & $\ldots$ & {$[74]$} \\
59 & 0.2 & $29.5 \pm 0.5$ & {$[1.5]$} & $\ldots$ & $\ldots$ & 0.07 & $\ldots$ & $\ldots$ & {$[75]$} \\
\hline
\end{tabular}

* Hydrogen column density (in units of $10^{22} \mathrm{~cm}^{-2}$ ) obtained from spectral fits to the PWN spectra or estimated from the pulsar's dispersion measure assuming $10 \%$ interstellar medium ionization (in square brackets for the latter case). In a few cases upper limits are given based on the galactic HI column density.

$\dagger$ Logarithm of PWN luminosity in the $0.5-8 \mathrm{keV}$ band. The quoted errors are purely statistical, they do not include the distance errors. For bright PWNe (e.g., \#\# 2, 8, 16), we quote the luminosity of the PWN "core" restricted to the torus/arcs regions. For the PWNe with extended tails (\#\# 39, 42, 48, 53, 54, 55, 58) we quote only the luminosity of the bright "bullet" component. For \#\# 36, 45 and 46, faint extended emission is seen around the pulsar but its luminosity is very uncertain; we use \pm 0.50 dex as a conservative estimate for the uncertainty.

** Logarithm of PWN (or candidate) luminosity in the 1-10 TeV band.

* Photon index of TeV spectrum determined from a power-law model. The fits are not good (e.g., an exponential cutoff is required or the spectral slope is nonuniform) in the cases marked by the superscript ${ }^{\text {? }}$. The values in square brackets were assumed for estimating $L_{\gamma}$.

$\S$ Size of X-ray PWN "core" in which the PWN X-ray properties listed in this table were measured.

II Size of TeV source. If the source is unresolved, we quote the upper limit on $l_{\gamma}$.

$\|$ Offset between the X-ray and TeV components.

$\dagger \dagger$ The PWN/PSR X-ray properties listed here were measured by ourselves (except for \#\# 2, 5, 6, 10, 37, 47, 49 and 51), but we cite recent relevant papers when available.

around the pulsar is considered. Also, the IC spectrum may deviate from a PL at large energies due to the intrinsic upper boundary of the electron SED, or due to the cooling effects. In addition to the spectral differences caused by the cooling and boundary effects, one should keep in mind that while the intensity of the synchrotron component depends on the magnetic field strength, the IC intensity depends on the ambient radiation density instead. Therefore, there is no reason to expect direct proportionality between the X-ray and TeV fluxes of a particular PWN, and indeed we see little correlation between the X-ray and TeV luminosities (Fig. 1, panel A). Yet, the X-rays, observed from the innermost PWN region, and $\mathrm{TeV} \gamma$-rays, produced throughout the entire PWN volume, can be related in a model that takes into account the pulsar wind history (e.g., the evolution of $\dot{E}$ and the PWN size), its spatially varying properties, and local radiation density. While measured from spatially different regions of a PWN, the X-ray and TeV luminosities in Table 2 still can be used to test such models as long as the observed values are compared with the model values calculated for appropriate PWN zones and time intervals. There have been a number of analytical models that take into account either the spatial dependence (e.g., [76, 77]) or time-dependence (e.g., [78, 79]) of the PWN properties but rarely both ([80]). 

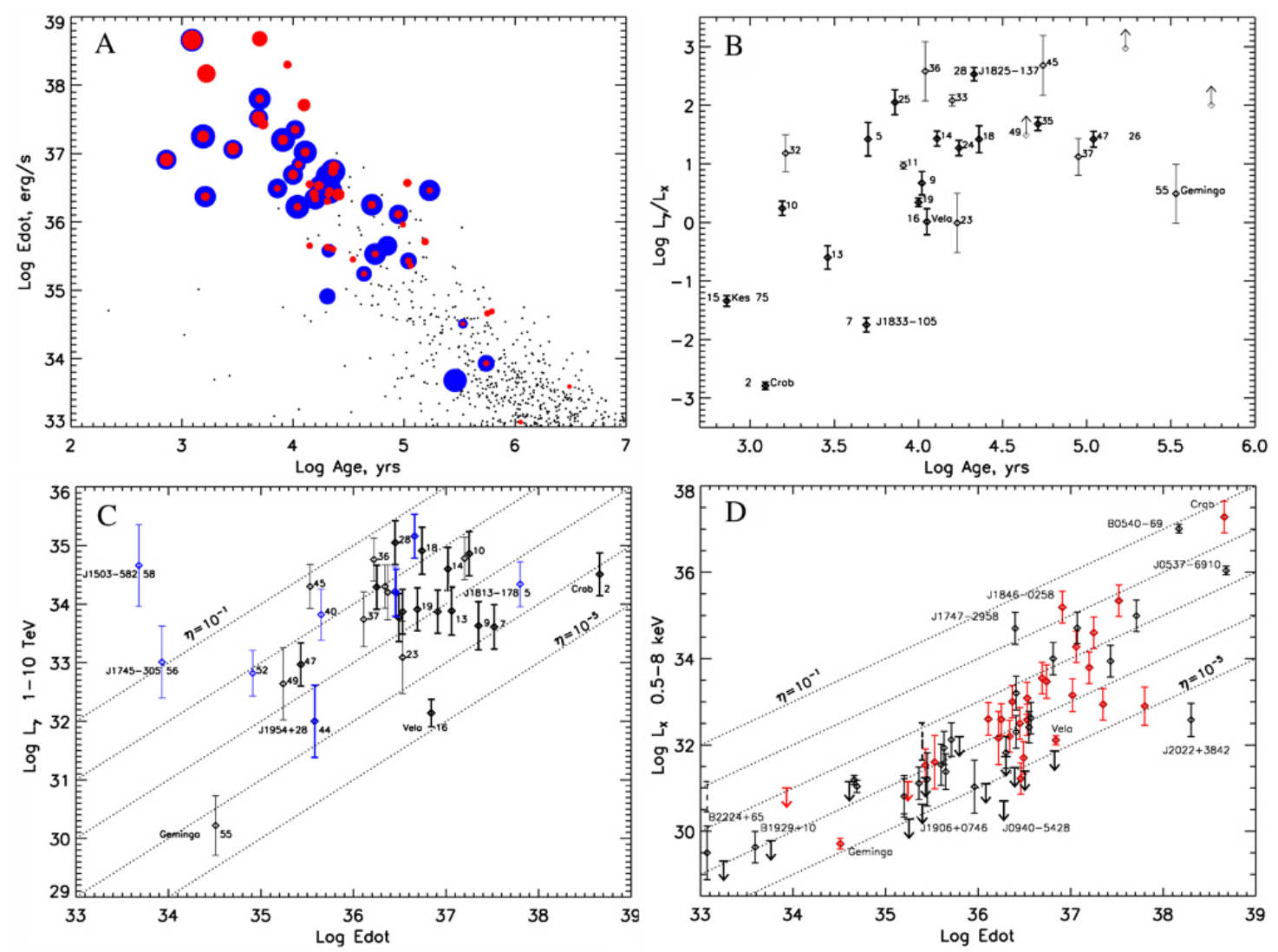

FIGURE 1. (A) Filled circles are X-ray (red) and TeV (blue) detected PWNe or PWN candidates. Larger circle sizes correspond to higher luminosities. The small black dots denote the pulsars from the ATNF catalog ([81]). (B) Ratio of the TeV to X-ray luminosities vs. pulsar's spin-down age. Here and in panel $C$ the thin error bars mark questionable associations. $(C) \mathrm{TeV}$ luminosities of PWNe and PWN candidates vs. pulsar's $\dot{E}$. The blue error bars mark TeV objects without X-ray counterparts. Here and in panel $D$ the luminosity uncertainties include $50 \%$ systematic uncertainty assigned to the distances unless the pulsar is in LMC or its parallax has been measured. $(D)$ X-ray luminosities of PWNe vs. $\dot{E}$. The red error bars denote X-ray PWNe with $\mathrm{TeV}$ counterparts. The lines of constant radiative efficiency $\left(\eta \equiv L_{X, \gamma} / \dot{E}\right)$ are plotted in panels $C$ and $D$.

In panels $\mathrm{C}$ and $\mathrm{D}$ of Figure 1 we show the dependences of the luminosities $L_{X}$ and $L_{\gamma}$ on $\dot{E}$. As expected, we do not see an obvious correlation between $L_{\gamma}$ and $\dot{E}$, which likely reflects the fact that the $\mathrm{TeV}$ luminosity depends on the history of the pulsar spin-down rather than on the current $\dot{E}$. An additional scatter is expected due to the differences in the local IR background and the uncertain distances. Most of the 1-10 $\mathrm{TeV}$ luminosities cluster in the range of $10^{33}-10^{35} \mathrm{erg} \mathrm{s}^{-1}$, a much narrower span than that attained by the X-ray luminosities shown in another panel. The $L_{X}$ versus $\dot{E}$ plot does show some correlation between the two; however, the more objects are included the larger the scatter becomes, weakening the correlation. It seems very unlikely that a factor of $10^{4}$ scatter at a given $\dot{E}$ could be explained by varying environment (e.g., ambient pressure) or differences in pulsar velocities. Some "hidden" pulsar parameters (e.g., the angle between the rotation and magnetic axis, or the topology of the NS magnetic field) may instead govern the efficiency of the magnetic-to-kinetic energy conversion and, as a result, the X-ray efficiencies of PWNe (see also [2]). In Figure 1 (panel B) we also 
plot the distance-independent $L_{\gamma} / L_{X}$ as a function of spin-down age $\tau$, which shows a hint of positive correlation between the two, at younger ages. This correlation can be explained by the correlation between $L_{X}$ and $\dot{E}$ (hence the anti-correlation between $L_{X}$ and $\tau$ ). These results are in general agreement with the recent findings of [82] based on a smaller sample of PWNe. At $\log \tau \gtrsim 4.5$ the dependence of $L_{\gamma} / L_{X}$ on $\tau$ appears to level off as expected from the simple model of [82]. This trend, however, shows a large scatter, which might be attributed to the magnetic and radiation fields being different for different objects.

To conclude, multiwavelength observations of PWNe are crucial because they provide identifications for VHE sources and reveal the true energetics and composition of pulsar winds. In addition to $\mathrm{X}$-ray and $\mathrm{TeV}$ observations, there is an opportunity to detect the IC PWN component with Fermi in GeV, where even old objects are expected to exhibit uncooled IC spectrum which should be more directly linked to the radio synchrotron component. The data accumulation must be complemented by development of multizone models of PWN evolution, essential for understanding the role of pulsar winds in seeding the Galaxy with energetic particles and magnetic fields.

\section{ACKNOWLEDGMENTS}

We would like to thank Wenwu Tian for providing updated distances for several sources. The authors acknowledge support from NASA grants NNX09AC81G and NNX09AC84G, and Chandra award AR8-9009X. This material is also based upon work supported by the National Science Foundation under Grants No. 0908733 and 0908611.

\section{REFERENCES}

1. B. M. Gaensler, and P. O. Slane, Ann. Rev. A\&A 44, 17-47 (2006).

2. O. Kargaltsev, and G. G. Pavlov, "Pulsar Wind Nebulae in the Chandra Era," in AIP Conf. Proc., edited by C. Bassa, Z. Wang, A. Cumming, \& V. M. Kaspi, 2008, vol. 983, pp. 171-185.

3. D. A. Leahy, and W. W. Tian, $A \& A$ 480, L25-L28 (2008).

4. Y. Su, Y. Chen, J. Yang, et al., ApJ 694, 376-386 (2009).

5. Y. Chen, Q. D. Wang, E. V. Gotthelf, et al., ApJ 651, 237-249 (2006).

6. K. Mori, D. N. Burrows, J. J. Hester, et al., ApJ 609, 186-193 (2004).

7. R. Willingale, B. Aschenbach, R. G. Griffiths, et al., A\&A 365, L212-L217 (2001).

8. F. Aharonian, et al., A\&A 457, 899-915 (2006).

9. Z. Arzoumanian, talk at Supernova Remnants and PWNe in the Chandra Era, Boston, MA (2009).

10. P. Kaaret, H. L. Marshall, T. L. Aldcroft, et al., ApJ 546, 1159-1167 (2001).

11. D. J. Helfand, E. V. Gotthelf, J. P. Halpern, et al., ApJ 665, 1297-1303 (2007).

12. F. Aharonian, et al., ApJ 636, 777-797 (2006).

13. E. V. Gotthelf, and J. P. Halpern, ApJL 700, L158-L161 (2009).

14. M. Renaud, V. Marandon, E. V. Gotthelf, et al., arXiv:0910.3074 (2009).

15. S. Safi-Harb, I. M. Harrus, R. Petre, et al., ApJ 561, 308-320 (2001).

16. A. Djannati-Atai, O. C. de Jager, R. Terrier, et al., "New Companions for the lonely Crab? " in International Cosmic Ray Conf. Proc., 2008, vol. 2, pp. 823-826.

17. P. Slane, D. J. Helfand, E. van der Swaluw, et al., ApJ 616, 403-413 (2004).

18. J. P. Halpern, F. Camilo, E. V. Gotthelf, et al., ApJL 552, L125-L128 (2001).

19. E. Aliu, talk at Supernova Remnants and PWNe in the Chandra Era, Boston, MA (2009).

20. B. M. Gaensler, J. Arons, V. M. Kaspi, et al., ApJ 569, 878-893 (2002).

21. F. Aharonian, et al., $A \& A$ 435, L17-L20 (2005). 
22. O. Kargaltsev, G. G. Pavlov, and J. A. Wong, ApJ 690, 891-901 (2009).

23. J. P. Hughes, P. O. Slane, D. N. Burrows, et al., ApJL 559, L153-L156 (2001).

24. F. J. Lu, Q. D. Wang, B. Aschenbach, et al., ApJL 568, L49-L52 (2002).

25. C.-Y. Ng, M. S. E. Roberts, and R. W. Romani, ApJ 627, 904-909 (2005).

26. F. Aharonian, et al., $A \& A$ 456, 245-251 (2006).

27. D. J. Helfand, B. F. Collins, and E. V. Gotthelf, ApJ 582, 783-792 (2003).

28. G. G. Pavlov, O. Y. Kargaltsev, D. Sanwal, et al., ApJL 554, L189-L192 (2001).

29. D. J. Helfand, E. V. Gotthelf, and J. P. Halpern, ApJ 556, 380-391 (2001).

30. F. Aharonian, et al., $A \& A$ 448, L43-L47 (2006).

31. M. S. E. Roberts, C. R. Tam, V. M. Kaspi, et al., ApJ 588, 992-1002 (2003).

32. E. V. Gotthelf, and J. P. Halpern, ApJ 681, 515-521 (2008).

33. J. W. T. Hessels, D. J. Nice, B. M. Gaensler, et al., ApJL 682, L41-L44 (2008).

34. F. Aharonian, et al., $A \& A$ 477, 353-363 (2008).

35. D.-S. Moon, J.-J. Lee, S. S. Eikenberry, et al., ApJL 610, L33-L36 (2004).

36. X. H. Li, F. J. Lu, and T. P. Li, ApJ 628, 931-937 (2005).

37. M. Roberts, C. Brogan, and M. Lyutikov, BAAS 38, 997 (2007).

38. J. W. T. Hessels, M. S. E. Roberts, S. M. Ransom, et al., ApJ 612, 389-397 (2004).

39. A. A. Abdo, et al., ApJL 700, L127-L131 (2009).

40. R. W. Romani, C.-Y. Ng, R. Dodson, et al., ApJ 631, 480-487 (2005).

41. S. Hoppe, E. de Oña-Wilhemi, B. Khélifi, et al., arXiv:0906.5574 (2009).

42. V. E. Zavlin, ApJL 665, L143-L146 (2007).

43. F. Aharonian, et al., $A \& A$ 484, 435-440 (2008).

44. F. Aharonian, et al., $A \& A$ 499, 723-728 (2009).

45. B. M. Gaensler, N. S. Schulz, V. M. Kaspi, et al., ApJ 588, 441-451 (2003).

46. G. G. Pavlov, O. Kargaltsev, and W. F. Brisken, ApJ 675, 683-694 (2008).

47. F. Aharonian, et al., $A \& A$ 460, 365-374 (2006).

48. V. M. Kaspi, E. V. Gotthelf, B. M. Gaensler, et al., ApJL 562, L163-L166 (2001).

49. F. Camilo, B. M. Gaensler, E. V. Gotthelf, et al., ApJ 616, 1118-1123 (2004).

50. B. M. Gaensler, E. van der Swaluw, F. Camilo, et al., ApJ 616, 383-402 (2004).

51. M. Gonzalez, and S. Safi-Harb, ApJL 591, L143-L146 (2003).

52. A. Djannati-Atai, talk at Supernova Remnants and PWNe in the Chandra Era, Boston, MA (2009).

53. O. Kargaltsev, G. G. Pavlov, and G. P. Garmire, ApJ 660, 1413-1423 (2007).

54. O. Kargaltsev, G. G. Pavlov, and G. P. Garmire, ApJ 670, 643-654 (2007).

55. M. E. Gonzalez, V. M. Kaspi, M. J. Pivovaroff, et al., ApJ 652, 569-575 (2006).

56. O. Kargaltsev, and G. G. Pavlov, ApJ 670, 655-667 (2007).

57. F. Aharonian, et al., $A \& A$ 472, 489-495 (2007).

58. F. Aharonian, et al., $A \& A$ 439, 1013-1021 (2005).

59. J. A. Hinton, S. Funk, S. Carrigan, et al., $A \& A$ 476, L25-L28 (2007).

60. O. Kargaltsev, Z. Misanovic, G. G. Pavlov, et al., ApJ 684, 542-557 (2008).

61. J. P. Halpern, E. V. Gotthelf, F. Camilo, et al., ApJ 612, 398-407 (2004).

62. R. Petre, K. D. Kuntz, and R. L. Shelton, ApJ 579, 404-410 (2002).

63. F. Camilo, P. S. Ray, S. M. Ransom, et al., ApJ 705, 1-13 (2009).

64. F. Aharonian, et al., A\&A 393, L37-L40 (2002).

65. R. Mukherjee, E. V. Gotthelf, and J. P. Halpern, Astrophys. Space Sci. 309, 29-33 (2007).

66. P. Weltevrede, A. A. Abdo, M. Ackermann, et al., ApJ 708, 1426-1441 (2010).

67. B. W. Stappers, B. M. Gaensler, V. M. Kaspi, et al., Science 299, 1372-1374 (2003).

68. N. Kawai, talk at Fermi Symposium, Washington, DC (2009).

69. O. Tibolla, R. C. G. Chaves, O. de Jager, et al., arXiv:0907.0574 (2009).

70. R. W. Romani, and C.-Y. Ng, ApJL 585, L41-L44 (2003).

71. C.-Y. Ng, R. W. Romani, W. F. Brisken, et al., ApJ 654, 487-493 (2007).

72. K. E. McGowan, W. T. Vestrand, J. A. Kennea, et al., ApJ 647, 1300-1308 (2006).

73. G. G. Pavlov, D. Sanwal, and V. E. Zavlin, ApJ 643, 1146-1150 (2006).

74. Z. Misanovic, G. G. Pavlov, and G. P. Garmire, ApJ 685, 1129-1142 (2008).

75. C. Y. Hui, and W. Becker, A\&A 467, 1209-1214 (2007).

76. C. F. Kennel, and F. V. Coroniti, ApJ 283, 694-709 (1984).

77. C. F. Kennel, and F. V. Coroniti, ApJ 283, 710-730 (1984). 
78. R. A. Chevalier, ApJL 539, L45-L48 (2000).

79. J. D. Gelfand, P. O. Slane, and W. Zhang, ApJ 703, 2051-2067 (2009).

80. E. Amato, M. Salvati, R. Bandiera, et al., A\&A 359, 1107-1110 (2000).

81. R. N. Manchester, G. B. Hobbs, A. Teoh, et al., AJ 129, 1993-2006 (2005).

82. F. Mattana, M. Falanga, D. Götz, et al., ApJ 694, 12-17 (2009). 